\title{
Clinical outcome in two adult horses treated for cleft palate with laryngeal tie-forward surgery
}

\author{
Irene Tosi, ${ }^{1}$ Sigrid Grulke, ${ }^{2}$ Alexandra Salciccia, ${ }^{2}$ Geoffroy de la Rebière de Pouyade ${ }^{2}$
}

'Department of Physiology and Equine Sports Medicine, Faculty of Veterinary Medicine, University of Liège, Liege, Belgium

${ }^{2}$ Department of Companion Animals and Equids, Faculty of Veterinary Medicine, University of Liège, Liège, Belgium

\section{Correspondence to} Irene Tosi; irene.tosi@uliege.be

Received 22 January 2018 Revised 30 April 2018 Accepted 13 May 2018
Check for updates

To cite: Tosi I, Grulke S, Salciccia A, et al. Vet Rec Case Rep Published Online First: [please include Day Month Year]. doi:10.1136/ vetreccr-2018-000605

\section{SUMMARY}

Two adult sport horses were referred for respiratory noise, exercise intolerance, coughing and nasal discharge containing food material. An asymmetrical, mild to moderate defect of the soft palate was diagnosed and surgically treated by laryngeal tie-forward procedure (LTFP). The immediate postoperative endoscopic images were satisfactory; the epiglottis was in normal alignment, dorsal to the soft palate defect in both cases. When contacted respectively four months and three years after surgery, owners of both horses declared being satisfied with the outcome of surgery as initial clinical signs had reduced or disappeared. Follow-up endoscopy showed some retraction of the larynx in both horses but the reduction of the palatal defect was adequate. This report demonstrates that horses can reach adulthood with certain soft palate defects and that severity of clinical signs varies between individuals. The authors suggest considering LTFP as an option in adult horses with smallto-moderate cleft palates causing clinical signs.

\section{BACKGROUND}

Cleft palate or palatoschisis, is a congenital disorder due to an incomplete closure of palatal folds during gestation. It is rare in horses and mainly affects the caudal portion of the soft palate. ${ }^{12}$ Severely affected foals (ie, large palatal defects) are generally referred for coughing and milk regurgitation from the nostrils after nursing, consequent failure of passive immunity transfer, malnutrition or aspiration pneumonia. ${ }^{3-5}$ In these cases survival rate is poor without surgery. Nonetheless, if the defect is small, some foals may reach maturity and may even be able to perform without displaying severe clinical signs and thus without a definitive diagnosis being established..$^{5-7}$ In severely affected foals primary cleft palate repair is considered a salvage procedure, due to incomplete development of the intrinsic palatal musculature and due to the high level of tension on primary surgical repairs. Primary surgical repair is associated to a poor postoperative prognosis due to a high complication rate. Difficult surgical access to the palate also contributes to the complication rate whether a transoral approach, mandibular symphysiotomy, laryngotomy or pharyngotomy is used. ${ }^{17}$ Common postsurgical complications include dehiscence of the repair, osteitis or osteomyelitis of the mandibular symphysis, aspiration pneumonia, oronasal fistulas and pharyngeal dysfunction that could preclude an athletic career. ${ }^{35}$ However, a few horses with cleft palate have been reported to race either after surgical or conservative treatment. ${ }^{6-8}$
The laryngeal tie-forward procedure (LTFP) is a surgical technique generally employed to treat intermittent dorsal displacement of the soft palate (DDSP). This technique advances and elevates the laryngeal position by placing sutures between the thyroid cartilage and the basihyoid bone. ${ }^{9}{ }^{10}$ The postoperative complication rate is less than $8 \%$ and includes suture breakage, cartilage failure, incisional seroma/swelling and potential vocal fold collapse. ${ }^{10-12}$ Application of LTFP for soft palate defects is poorly documented. In a recent publication $^{6}$ this technique was used in a four-year-old riding pony with a mild and symmetrical soft palate defect. A marked reduction of the defect was seen immediately after surgery but recurrence of pharyngeal contamination by food was found nine months after surgery. The objective of this report was to present the clinical outcome of two adult sport horses diagnosed with a mild to moderate and asymmetrical defect of the caudal border of the soft palate which was treated by LTFP.

\section{CASE PRESENTATION}

Case 1 was an eight-year-old crossbred gelding, used for low-level riding (flat work and jumping over $80 \mathrm{~cm}$ ), presented for respiratory noise at work, nasal discharge containing food material and coughing at rest and during exercise. The clinical signs started shortly after the horse was purchased while it was being broken in. On field, resting endoscopy performed by the referring veterinarian had shown a permanent DDSP and an abnormal morphology of the soft palate.

Case 2 was an eight-year-old Holstein mare, used for intermediate-level riding (flat work and jumping over $120 \mathrm{~cm}$ ), presented in October 2016 for respiratory noise, coughing both at rest and during exercise, nasal discharge, exercise intolerance and difficulty in gaining weight. The mare had been purchased two years before the date of referral and its previous history was unknown.

\section{INVESTIGATIONS}

On presentation, case 1 had a good body condition score $(565 \mathrm{~kg}$, body condition score (BCS) $5 / 9$ according to the Henneke system) and a normal physical examination except for a bilateral mucopurulent nasal discharge mixed with food particles. Bronchopulmonary auscultation was normal. Complete blood count (CBC) including leucocyte differential count (LDC) on venous blood was normal. During the upper respiratory tract 


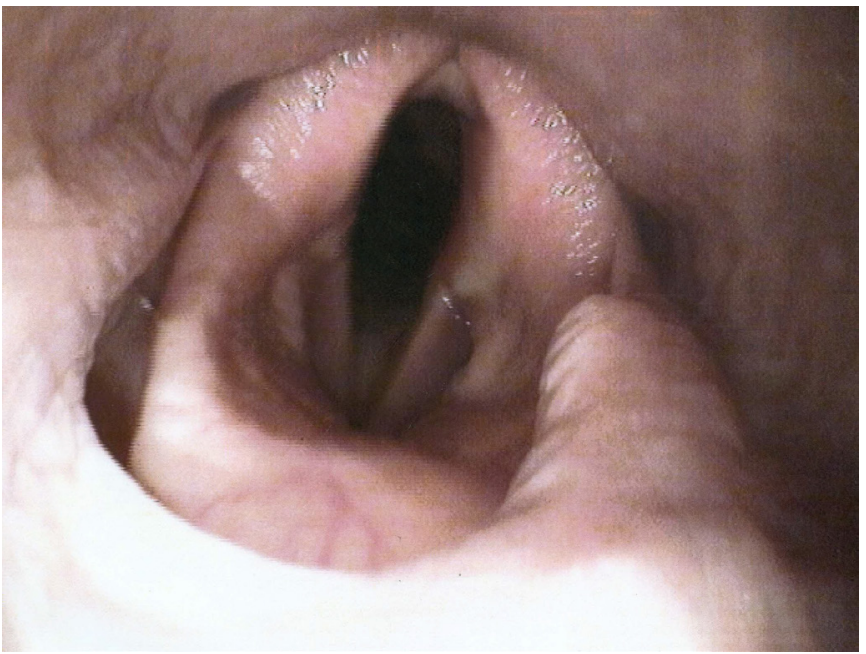

FIG 1: Resting preoperative upper airway endoscopy of case 1. This gelding was presented for respiratory noise, nasal discharge containing food material and coughing at rest and during exercise. The caudal border of the soft palate is incomplete and asymmetrical (the defect is wider on the right side). The caudal pillars of the soft palate look underdeveloped.

endoscopy, several clumps of food were noticed in the nasal cavities and pharynx; the caudal portion of the soft palate was asymmetrical and incomplete mostly on the right side (Fig 1), caudal pillars of the soft palate looked underdeveloped. A small fold of thickened mucosa was also visible on the caudal border of the soft palate on the left side. The DDSP was persistent, not resolving with deglutition. Tracheal wash was not performed in this horse.

At presentation, case 2 was considered moderately thin (496 kg, BCS 4/9). Physical examination was normal except for a bilateral mucous nasal discharge and submandibular lymphadenopathy (right lymph node was bigger than the left one).

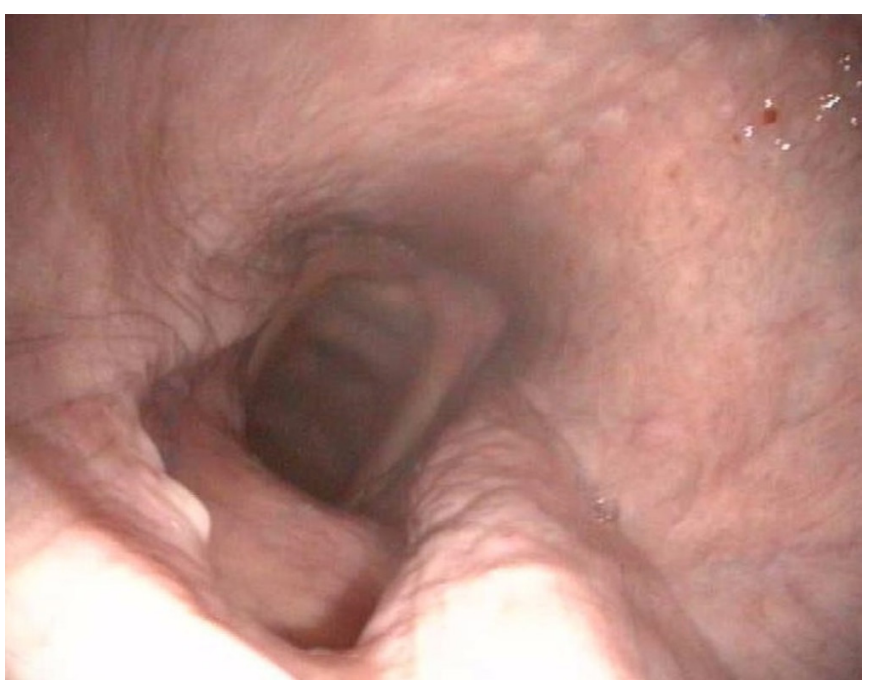

FIG 2: Resting preoperative upper airway endoscopy of case 2. The mare was presented for respiratory noise, coughing, nasal discharge, exercise intolerance and difficulty in gaining weight. There is an asymmetrical defect in the caudal part of the soft palate that is wider on the right side but longer on the left side. Caudal pillars of the soft palate are above the borders of the epiglottis and the former is dropped down into the oral cavity.
Bronchopulmonary auscultation revealed increased respiratory sounds but no crackles or wheezes. CBC and LDC on venous blood and arterial gas analysis were normal. Resting upper respiratory tract endoscopy revealed the presence of food particles in the nasal cavities and pharynx, and an asymmetrical defect in the caudal part of the soft palate. This defect was longer on the left side but wider on the right side (Fig 2). The caudal pillars of the soft palate were above and not behind the lateral borders of the epiglottis. There was constant communication with the oral cavity and the epiglottis was lying within it. Other abnormalities noted were lymphoid pharyngeal hyperplasia score III/ IV and a moderate dorsoventral nasopharyngeal collapse. The proximal trachea was covered by mucous secretions (score III/V) mixed with food particles. Bacteriology from the tracheal wash was indicative of infection by Escherichia coli. Degenerated neutrophils (65\% of the total cell count), food material, free and intracellular bacteria were also visible at the microscopic analysis. The final diagnosis in both horses was cleft palate with consequent lower airway contamination. The defect was judged asymmetrical and mild to moderate, ${ }^{6}$ even if it was difficult to score it objectively due to its asymmetry. In both cases the soft palate was over and not under the epiglottis. Asymmetry and width of the defects ruled out primary cleft palate repair as a surgical option due to the high risk of dehiscence. Elevating and advancing the larynx to limit the gap between the caudal aspect of the soft palate and the edge of the epiglottis was considered a better approach. As a consequence, LTFP was proposed and accepted by each owner.

\section{TREATMENT}

The preoperative care and the surgical procedure were identical in both horses. They received systemic routine perioperative antimicrobials and anti-inflammatory drugs. Food was witheld for 12 hours before surgery. A catheter was placed aseptically in the jugular vein. Premedication was achieved with $0.1 \mathrm{mg} / \mathrm{kg}$ intramuscular acepromazine, then with $0.6 \mathrm{mg} / \mathrm{kg}$ intravenous xylazine and induction was performed with a combination of $0.06 \mathrm{mg} / \mathrm{kg}$ intravenous midazolam and $2.2 \mathrm{mg} / \mathrm{kg}$ intravenous ketamine. After endotracheal intubation, horses were placed in dorsal recumbency and anaesthesia was maintained with isoflurane vaporised in oxygen and air through a circle anaesthetic system (Tafonius Wind God, Hallowell EMC, Pittsfield, Massachusetts, USA). Surgery was performed as described by Cheetham and colleagues ${ }^{13}$ using a modified technique of the original one described by Ducharme and colleagues. ${ }^{9}$ Briefly, after aseptic preparation and skin incision (starting caudally to the cricoid cartilage and extending rostrally to the caudal aspect of the basihyoid bone), the ventral aspect of the larynx was exposed by dividing the paired sternohyoid muscles. A No. 5 United States Pharmacopeia (USP) polyblend suture (Fiberwire) was passed twice through one wing of the thyroid cartilage ventral to the sternothyroid muscle, thus forming a loop in the thyroid lamina in such a way as to increase the strength of the fixation by distributing the tensions throughout the cartilage. ${ }^{10}$ The procedure was repeated on the other side. The ipsilateral dorsal and the contralateral ventral sutures were passed dorsally to the basihyoid bone on either side of the lingual process. A bilateral partial sternothyroidectomy was performed at this moment. Sutures were then tied independently using a slip knot with the head of the horse lifted to form a $90^{\circ}$ angle with the neck. Therefore, the rostral aspect of the thyroid cartilage was located immediately dorsal and rostral to the caudal border of the basihyoid bone. The subcutaneous tissues and skin were then closed in a routine manner. 


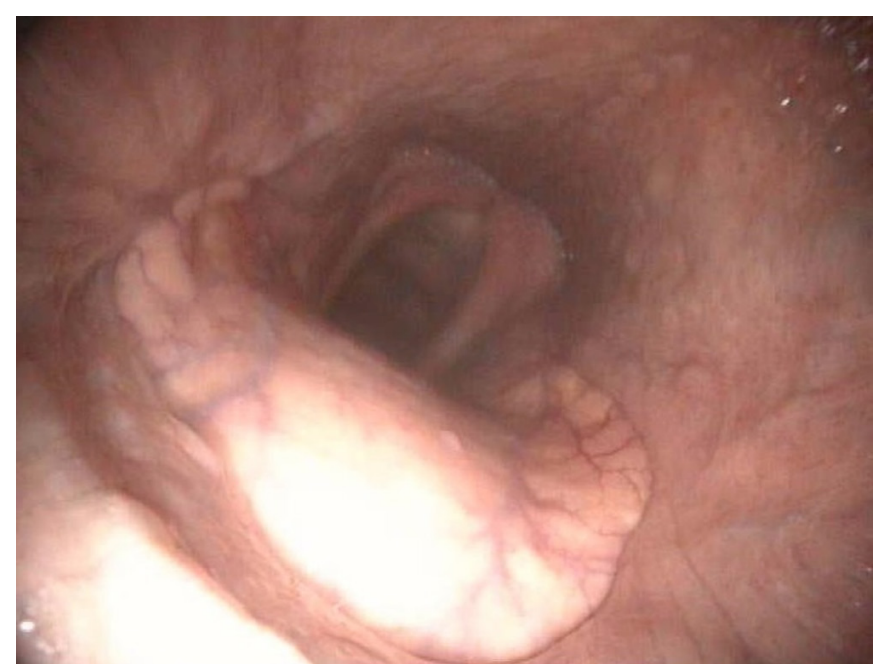

FIG 3: Resting postoperative upper airway endoscopy of case 2 before being discharged from the clinic. The larynx has moved dorsally and rostrally so as to restore a normal anatomical alignment between the epiglottis and the soft palate; the defect is no longer visible. The edge of the epiglottis is in close contact with the caudal border of the soft palate. The caudal pillars of the soft palate are now hidden behind the sides of the epiglottis.

\section{OUTCOME AND FOLLOW-UP \\ Postoperative evaluation}

Both horses recovered from surgery without complications. Hay and water were provided at shoulder height. In the immediate postoperative period the nasal discharge decreased quickly in both horses and no cough was noted at rest. A resting endoscopy was repeated before discharging the two horses from the clinic. In both cases, the larynx was located in a more dorsal and rostral position than before surgery, with the margins of the epiglottis covering the defect of the soft palate and being above and in close contact with its caudal border (Fig 3). Food particles were no longer visible in the nasal cavities and pharynx and no DDSP was noted even after deglutition. Horses were discharged from the clinic with instructions for aftercare.

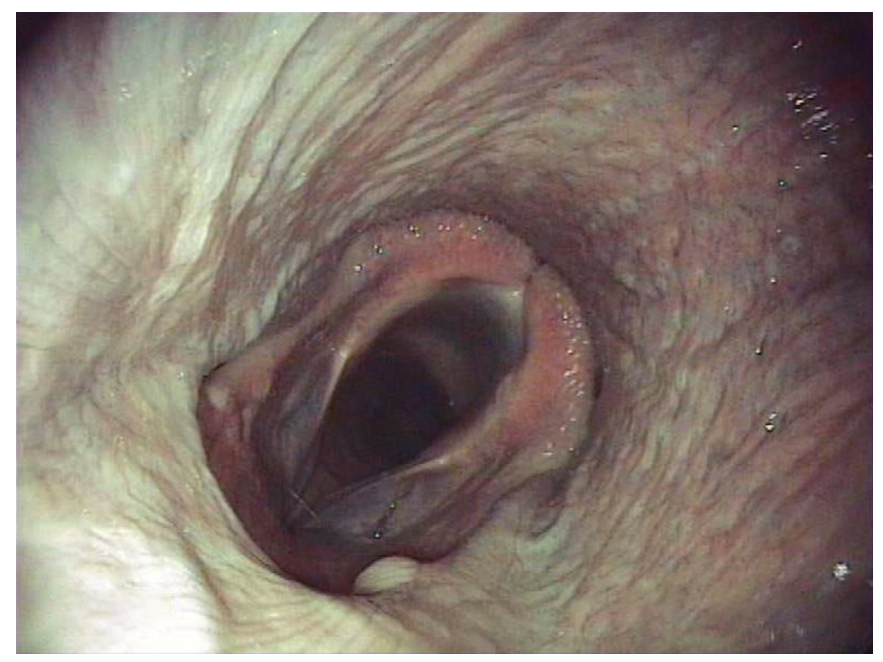

FIG 4: Resting upper airway endoscopy of case 1 performed three years after tie-forward surgery. There is still a small gap between the soft palate and the edge of the epiglottis. There is a small thickened fold of palatal mucosa on the left side of the soft palate that was also visible before surgery.

\section{Follow-up}

Owners were contacted by telephone in February 2017, respectively, three years and four months after surgery for cases 1 and 2. Owners were asked if they were satisfied with the intervention as well as to report on complications and on the performance of their horse (if improved, unchanged or worsened). They were asked if initial clinical signs had disappeared or persisted, and in that case, if it had waxed or waned. A follow-up examination and endoscopy at their stables were performed. In both cases, the owners reported that they were satisfied with the outcome of the intervention. In case 1, the nasal discharge and respiratory noise had completely disappeared. Coughing had disappeared for approximatively six months then it had reappeared, even if only limited to the onset of exercise. Managing bedding conditions (with shavings instead of straw) had seemed to reduce the coughing. The horse was performing at the same level of exercise as before surgery but due to the owner's choice and not because of lack of ability. At the follow-up visit, physical examination was normal apart from some bilateral mucoid nasal discharge. Respiratory auscultation was normal. The upper respiratory endoscopy showed that the larynx had partially retracted (Fig 4 ) in comparison to the immediate postoperative image, but the gap was still subjectively smaller than before surgery. There was an intermittent DDSP promptly resolving with deglutition movements. The small thickened fold of palatal tissue on the caudal border of the soft palate was still present and more easily visible. Contamination with only one or two small patches of food was observed in the upper respiratory tract of this horse (nasal cavities, pharynx and trachea). Tracheal secretions were seromucoid in nature and were scored III/V in quantity. A tracheal wash was performed and the cytological analysis revealed neutrophilic inflammation but no trace of food particles or bacteria at microscopy.

Concerning case 2 , the mare had gained weight with its BCS changing from $4 / 9$ to $5 / 9$. It was performing better than before surgery and was now jumping courses of $120 \mathrm{~cm}$ to $145 \mathrm{~cm}$ in height. The cough and nasal discharge had disappeared. While the respiratory noise observed during exercise had persisted, it was less audible than before surgery. At the follow-up visit, physical examination was normal. Submandibular lymph nodes were of normal size, and minimal mucoid nasal discharge was present. As in case 1 , follow-up upper respiratory endoscopy revealed a more caudal position of the larynx in comparison to the immediate postoperative image, but again, the gap between the border of the soft palate and the edge of the epiglottis was subjectively found smaller than before surgery (Fig 5). The caudal pillars of the soft palate were in close contact with the borders of the epiglottis. The epiglottis was lying immediately caudal to the soft palate and not ventral to it and within the oropharynx as before surgery. The proximal trachea was cleaner in comparison to the preoperative image, only two spots of food particles were seen in the very proximal trachea. The tracheal mucus was scored III/V; the tracheal wash was repeated and no bacteria or food particles were seen during the microscopic examination. Epithelial cells and less than $20 \%$ of non-degenerate neutrophils were noted.

\section{DISCUSSION}

Cleft palate is a rare congenital deformity affecting $0.1 \%$ to $0.2 \%$ of the equine population, more commonly involving the caudal half or three quarters of the soft palate. ${ }^{14}$ Affected horses are generally identified quite early after birth, but it has been observed that some subjects can reach maturity displaying diverse clinical signs not necessarily correlated with the severity of the 


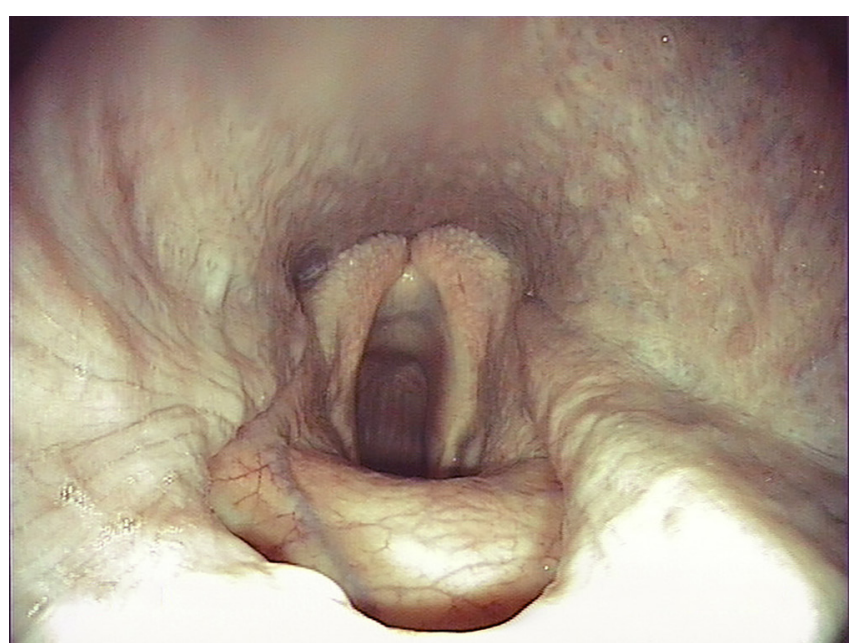

FIG 5: Resting upper airway endoscopy of case 2 performed four months after tie-forward surgery. The larynx has slightly moved backwards and the edge of the epiglottis is just caudal to the defect in the soft palate. The caudal pillars of the soft palate are in an intermediate position when compared with the preoperative and the immediate postoperative endoscopic appearance relative to the sides of the epiglottis. The former looks more dorsally positioned than before surgery.

defect. ${ }^{67}$ This report provides further evidence that some horses with a cleft palate can reach adult age with minimal morbidity. Horses described here were able to sustain physical activity, even if with some complaints.

Presence of food contamination in the upper respiratory tract and trachea was common to both cases but did not seem to have severe repercussions (ie, pneumonia) on the lower respiratory tract nor to cause clinical signs other than occasional coughing. Both horses were presented with respiratory noise during exercise. Unfortunately, an exercising endoscopy was not performed in any of these cases because of technical or economic reasons. Considering the unstable relationship between the epiglottis and the soft palate in both cases it seems logical to assume that the dorsal position of the soft palate relative to the epiglottis was the most likely cause of noise, even if other concurrent abnormalities (ie, intermittent epiglottic entrapment, axial deviation of the aryepiglottic folds, nasopharyngeal dynamic collapse, etc) as observed elsewhere ${ }^{6}$ could not be excluded.

It has been suggested that horses with small palatal defects can live and even perform without intervention. ${ }^{6-8}$ The decision to perform a surgical reduction was based on the owner's requirements for athleticism from their horses. Primary cleft palate repair was quickly excluded as a potential surgical approach because the defect was asymmetrical and located at the caudal edge of the soft palate. This part of the palate is a common site of repair failure because of inadequate surgical exposure and a lack of sufficient palatal tissue compared with the cranial portion. ${ }^{347}$ In cases like the ones the authors describe, where the defects are not extensive in length but are large in width, with more than $20 \%$ of palatal tissue missing, primary cleft palate repair is generally not recommended. ${ }^{14}$ LTFP represents a less invasive technique than primary cleft palate repair and is associated with fewer postoperative complications. To the authors' knowledge, LTFP has only been described once in the literature for cleft palate correction. It was performed on a four-year-old riding pony with a mild symmetrical defect of the soft palate. ${ }^{6}$ As in the present cases, the authors describe an initial improvement of the endoscopic appearance and a rapid reduction of the nasal discharge. However, a caudal movement of the larynx was observed nine months after surgery with persistence of food contamination of the nasopharynx and trachea despite this reduction of the nasal discharge. ${ }^{6}$ No information on the athletic outcome of the pony is available. In the present cases, both horses showed a caudal laryngeal retraction at the latest follow-up endoscopy and a gap between the caudal border of the defective soft palate and the epiglottis had reappeared. Nonetheless, the gap was judged subjectively smaller than before surgery and the owners had observed an improved athletic capability of their horses as well as a reduction or disappearance of the initial clinical signs. Moreover, minimal food contamination (limited to few and isolated flecks) was visible in the respiratory tract of both horses without microscopic evidence of bacterial infection in the tracheal wash. This corroborates with a recent suggestion that LTFP can improve deglutition and reduce aspiration in some models of pharyngeal dysphagia. ${ }^{15}$ It can be hypothesised that the observed caudal retraction of the larynx could be related to some degree of suture loosening occurring with time. In a similar manner, in prosthetic laryngoplasties, a 'relaxation' of the suture followed by some degree of loss of arytenoid abduction is frequently observed during the first four to six weeks after surgery. ${ }^{16} 17$ The cause of this phenomenon is still unclear, with cartilage or suture failure being two commonly postulated reasons. ${ }^{11}$ The two horses were checked endoscopically three years and four months postsurgery, respectively, and the caudal laryngeal retraction was visible on both animals. This suggests that this potential suture relaxation may occur early after surgery and likely remains unchanged after the first weeks, as observed in laryngoplasties. ${ }^{16}$ Unfortunately radiography of the larynx was not performed before or after surgery. It would have been interesting to measure the degree of caudal laryngeal retraction and how it evolved with time.

Retrospectively, the small mucosal fold on the caudal border of the soft palate in case 1 should have ideally been resected as these small masses can sometimes trigger DDSP and may account for the intermittent DDSP observed at the follow-up endoscopy. As already mentioned, a dynamic endoscopy would have been interesting so as to determine the exact cause of the respiratory noise observed in the two cases.

In conclusion, this report gives further confirmation that some horses can reach adulthood with certain palatal defects without severe clinical signs. Given all the complications associated with primary cleft palate repair, LTFP is a technique that should be considered as a surgical option to treat all adult horses with mild to moderate defects of the soft palate, either symmetrical or not, to reduce clinical signs arising from this condition.

Contributors IT examined the horses, performed the clinical and endoscopic examinations, the diagnosis, the follow up and wrote the paper. SG, AS and GdlRdP performed the surgical procedure and revised the paper. GdIRdP gave the idea to write this case report.

Funding The authors have not declared a specific grant for this research from any funding agency in the public, commercial or not-for-profit sectors.

Competing interests None declared.

Provenance and peer review Not commissioned; externally peer reviewed.

Data sharing statement All available data can be obtained from the corresponding author. 
(c) British Veterinary Association (unless otherwise stated in the text of the article) 2018. All rights reserved. No commercial use is permitted unless otherwise expressly granted.

\section{REFERENCES}

1 Semevolos SA, Ducharme N. Surgical repair of congenital cleft palate in horses: eight cases (1979-1997). AAEP Proceedings 1998;44:267-8.

2 Gaughan EM, DeBowes RM. Congenital diseases of the equine head. Vet Clin North Am Equine Pract 1993;9:93-110.

3 Bowman KF, Tate LP, Evans LH, et al. Complications of cleft palate repair in large animals. J Am Vet Med Assoc 1982;180:652-7.

4 Kirkham LE, Vasey JR. Surgical cleft soft palate repair in a foal. Aust Vet J 2002:80:143-6.

5 Sullivan EK, Parente EJ. Disorders of the pharynx. Vet Clin North Am Equine Pract 2003;19:159-67.

6 Barakzai SZ, Fraser BS, Dixon PM. Congenital defects of the soft palate in 15 mature horses. Equine Vet J 2014;46:185-8.

7 Murray SJ, Elce YA, Woodie JB, et al. Evaluation of survival rate and athletic ability after nonsurgical or surgical treatment of cleft palate in horses: 55 cases (19862008). J Am Vet Med Assoc 2013;243:406-10.

8 Haynes PF, Qualls CW. Cleft soft palate, nasal septal deviation, and epiglottic entrapment in a thoroughbred filly. J Am Vet Med Assoc 1981;179:910-3.
9 Ducharme NG, Hackett RP, Woodie JB, et al. Investigations into the role of the thyrohyoid muscles in the pathogenesis of dorsal displacement of the soft palate in horses. Equine Vet J 2003;35:258-63.

10 Woodie JB, Ducharme NG, Kanter P, et al. Surgical advancement of the larynx (laryngeal tie-forward) as a treatment for dorsal displacement of the soft palate in horses: a prospective study 2001-2004. Equine Vet J 2005;37:418-23.

11 Ahern BJ, Parente EJ. Surgical complications of the equine upper respiratory tract. Vet Clin North Am Equine Pract 2008;24:465-84.

12 Dart AJ. Vocal fold collapse after laryngeal tie-forward correction of dorsal displacement of the soft palate in a horse. Vet Surg 2006;35:584-5.

13 Cheetham J, Pigott JH, Thorson LM, et al. Racing performance following the laryngeal tie-forward procedure: a case-controlled study. Equine Vet J 2008;40:501-7.

14 Jones RS. Surgical repair of cleft palate in the horse. Equine Vet J 1975;7:86-90.

15 Virgin JE, Holcombe SJ, Caron JP, et al. Laryngeal advancement surgery improves swallowing function in a reversible equine dysphagia model. Equine Vet J 2016;48:362-7.

16 Brown JA, Derksen FJ, Stick JA, et al. Effect of laryngoplasty on respiratory noise reduction in horses with laryngeal hemiplegia. Equine Vet J 2004;36:420-5.

17 Dixon RM, McGorum BC, Railton DI, et al. Long-term survey of laryngoplasty and ventriculocordectomy in an older, mixed-breed population of 200 horses. Part 1: Maintenance of surgical arytenoid abduction and complications of surgery. Equine Vet J 2003;35:389-96.

Copyright 2018 British Veterinary Association. All rights reserved. For permission to reuse any of this content visit

http://www.bmj.com/company/products-services/rights-and-licensing/permissions/

Veterinary Record Case Reports subscribers may re-use this article for personal use and teaching without any further permission.

Subscribe to Vet Record Case Reports and you can:

- Submit as many cases as you like

- Enjoy fast sympathetic peer review and rapid publication of accepted articles

- Access all the published articles

- Re-use any of the published material for personal use and teaching without further permission

For information on Institutional Fellowships contact consortiasales@bmjgroup.com

Visit vetrecordcasereports.bvapublications.com for more articles like this and to become a subscriber 